\title{
Detection of Haemoparasites of Blood Donors in 9 Locations in and Around Plateau State, Nigeria
}

\author{
Mabel .E. Ike ${ }^{1} \quad$ Emmanuel . C. Onuoha ${ }^{2 *} \quad$ Asabe. J. Yohanna ${ }^{1} \quad$ Anthony. D. Dakul $^{1} \quad$ Garba .J. Damen $^{3}$ \\ Ezekiel. F.Hallie ${ }^{5}$ Vivian. A. Maduka ${ }^{2}$ Nake. Nelson-Ebimie ${ }^{2}$ \\ Jangfa. N. Nden ${ }^{2} \quad$ Tracy .A. Diepreye ${ }^{4}$ \\ 1. Department of Zoology, Faculty of Natural Sciences, University of Jos, Nigeria \\ 2. Department of Medical Laboratory, Federal University, Otuoke, Bayelsa State, Nigeria \\ 3. Department of Medical Laboratory Sciences, Faculty of Medical Sciences, University of Jos, Nigeria \\ 4. Pharmacy Unit, Medical Service Department, Federal University, Otuoke, Bayelsa State, Nigeria \\ 5. School of Pharmacy, University of Liberia, Monrovia, Liberia
}

\begin{abstract}
Haemoparasites in the tropics are also endemic in Nigeria. Asymptomatic infections may abound, due to resistance to these infections. This asymptomatic infection has been one of the factors, which has maintained transmission of these pathogens, through many ways, including blood donation and transfusion. In this report, haemoparasitic infections in blood donors have been described, from blood donors within Plateau State, Nigeria. Five hundred and twelve blood donors were selected by means of a random sampling method and their blood samples collected. Serological assay was done using rapid test kits to check for presence of antibodies (in the case of microfilariae) or antigens (in the case of malaria) to the different haemoparasites. Also, Elisa technique was used for the microfilariae. Thick and thin films were made from each blood sample on grease-free slides allowed to dry and stained by $3 \%$ Giemsa solution for $45 \mathrm{~min}$ which is the Giemsa technique. Results indicate that $270(52.7 \%)$ of the sample population had no infection; 121 ( 23.6\%) of the population were infected with Plasmodium falciparum; 11 (2.1\%) were infected with Plasmodium malariae; 69 (13.5\%) were infected with HBsAg; 29 (5.7\%) were infected with HCV; 7 ( I.4\% ) were infected with Trypanosoma brucei gambiense; 1\% were infected with microfilariae, $4(0.8 \%)$ of the $1 \%$ were unsheathed and identified to be Mansonella perstans, while $1(0.2 \%)$ were sheathed and identified to be Loa loa. Most blood group types were susceptible to haemoparasitic infections. The result of the study therefore stresses the need to screen blood for haemoparasites before transfusion, owing to the dangers of doing otherwise. The occupations and dwelling places of the donors are predisposing factors to these haemoparasitic infections. Since they have the passion to save lives through blood donation, they should therefore make the necessary adjustments that will make them more suitable lifesavers. It is recommended that the basic transmission factors of these parasites are explained to donors to reduce further incidences.
\end{abstract}

DOI: $10.7176 / \mathrm{JBAH} / 9-22-01$

Publication date: November $30^{\text {th }} 2019$

\section{Introduction}

Blood transfusion is considered a life-saving intervention that has an essential role in patient management within health care systems. All Member States of the World Health Organization (WHO) endorsed World Health Assembly resolutions [1] and [2] in 2005.

The establishment of systems, (like National Blood Transfusion Service in Nigeria), that ensure that all donated blood is screened for transfusion-transmissible infections is a core component of every national blood programme. Globally, however, there are significant variations in the extent to which donated blood is screened, the screening strategies adopted and the overall quality and effectiveness of the blood screening process. As a result, in many countries the recipients of blood and blood products remain at unacceptable risk of acquiring lifethreatening infections that could easily be prevented $[2,3]$.

The range of investigations carried out for donors need to be increased in practice, since transfusion reactions still occur despite complete cross-match of donor cells and recipient serum. This suggests the presence of some antigens which recipient develops antibodies to, hence a reaction. Not all reactions are therefore elicited as a result of incompatibility of the blood being given [4]. Haemoparasite infections are some of the commonest and most important diseases (antigens) of man in the tropics and subtropics. These infections are an important constraint to man since it affects man socially, economically and otherwise [5].

The aim of this study is to detect haemoparasites of among blood donors recruited from 9 locations in and around plateau state, Nigeria.

\section{Materials and Methods} 2.1 Study Area/Sample Size

The study was carried out in Jos, Plateau state and environs. Five hundred and twelve donors were selected by means of a random sampling method and their blood samples collected. Analyses were made for haemoparasites. 
The blood groups was also determined and recorded.

Prior to sample collection, an ethical clearance was obtained from NBTS ethical committee and oral consent was obtained from the donors before their names were inputted into the detail collection form.

\subsection{Sample Collection}

Blood samples were collected using sterile EDTA. This was achieved by tying a tourniquet above a prominent vein, and a sterile swab used to clean the area to reduce the risk of contamination. With a sterile syringe, blood is collected from the vein and immediately put into an EDTA bottle. The blood was mixed gently to avoid coagulation. All samples collected were properly labeled, kept in ice and transported to the Angel of Hope Clinic or DEE Medical Centre laboratory for processing within 3 hours of collection.

\subsection{Processing of Samples}

Serological assay was done using rapid test kits to check for presence of antibodies to (in the case of other haemoparasites) or antigens of (in the case of malaria parasites) the different haemoparasites. The various tests were run following manufacturer's guidelines/instructions.

Microfilaria was screened for using ELISA - Og4C3 monoclonal antibody assay, which is quantitative. The Leishmania and Trypanosoma serology kit was not readily available for this research.

Tests are positive for the particular blood parasite analyzed, if a double band was seen (for the rapid test kits) and agglutination or adhesion (for the ELISA). It was however confirmed using other test methods like microscopy, which has remained a standard for malaria and diagnosis of other haemoparasites [6].

Parasitological examination: Thick and thin films were made in duplicates from each blood sample on greasefree slides, allowed to dry and stained by $3 \%$ Giemsa solution for $45 \mathrm{~min}$ which is the Geimsa technique [6].

Stained blood films were examined under x100 objective lens of a microscope with the aid of immersion oil for haemoparasites. Thin films were used for the species identification of parasites. Further confirmation of positive samples were undertaken to ensure quality control.

Wet preparation and Delafield's haemalum Staining technique for microfilaria: This stain is a derivative of Haematoxylin and alum. It stains the nuclear matter. Eosin is used with it as a counter stain. The staining procedure was carried out according to the methods of Cheesbrough [6].

Determination of ABO blood group: Three spots of blood from each subject were made on the white plain tile and a drop of each antiserum A, B and D was applied to each of the different spots of blood, respectively.

The mixture was further stirred with a plastic stirrer and rocked for 2 minutes. A sign of agglutination on any of the spots was observed and recorded [6].

Statistical Analysis

Data obtained were subjected to statistical analyses (using mean of occurrence, percentage prevalence and Non Parametric Chi-Square test).

\section{Results}

Table 1: Distribution of the Parasites Among the Different Blood Group Types of Donors

\begin{tabular}{|c|c|c|c|c|c|c|c|c|c|}
\hline \multirow[b]{2}{*}{ PARASITES } & \multicolumn{9}{|c|}{ NUMBER INFECTED } \\
\hline & $\mathbf{A}+$ & A- & B+ & B- & $\mathbf{A B}+$ & AB- & O+ & O- & TOTAL \\
\hline P. falciparum & 19 & 0 & 33 & 3 & 4 & 0 & 59 & 3 & 121 \\
\hline P. malariae & 1 & 0 & 2 & 0 & 1 & 0 & 7 & 0 & 11 \\
\hline L. loa & 0 & 0 & 0 & 0 & 0 & 0 & 1 & 0 & 1 \\
\hline M. perstans* & 1 & 0 & 0 & 0 & 1 & 0 & 2 & 0 & 4 \\
\hline T. brucei gambiense & 3 & 0 & 1 & 0 & 0 & 0 & 3 & 0 & 7 \\
\hline HBsAg & 20 & 0 & 18 & 1 & 1 & 0 & 27 & 2 & 69 \\
\hline $\mathrm{HCV}$ & 8 & 0 & 5 & 0 & 2 & 0 & 14 & 0 & 29 \\
\hline TOTAL/ $\chi^{2}$ & $52 / 44.462^{\mathrm{a}}$ & 0 & $59 / 63.288^{\mathrm{a}}$ & $4 / 1.000^{\mathrm{a}}$ & $9 / 3.778^{a}$ & 0 & $113 / 163.841^{\mathrm{a}}$ & $5 / .200^{\mathrm{a}}$ & 242 \\
\hline
\end{tabular}

This table indicates the parasite spectrum in relation to blood group types. This is however a bit skewed and not holistic, since all blood group types was not available for the donation.

For those that however donated, Blood group O positive was highest for all parasite types. This was followed by Blood group B positive, then Blood group A positive. There were however cases where Blood group A positive recorded more infection than Blood group B positive (reference). It is however important to note that no matter the blood group, parasitaemia is possible. This means that every blood group is susceptible to blood parasitic infections. 
Table 2: Incidence of Haemoparasites among Blood Donors

\begin{tabular}{lll} 
PARASITE & No (\%) POSITIVE & VALID PERCENTAGE \\
\hline P. falciparum & $121(23.6)$ & 50 \\
Plasmodium malariae & $11(2.1)$ & 4.5 \\
sheathed Microfilaria (L. loa) & $1(0.2)$ & 0.4 \\
unsheathed Microfilaria (M. perstans) & $4(0.8)$ & 1.7 \\
T. brucei gambiense & $7(1.4)$ & 2.9 \\
HBsAg & $69(13.5)$ & 28.5 \\
HCV & $29(5.7)$ & 12 \\
Total infected & $242(47.3)$ & 100 \\
No infection & $270(52.7)$ & \\
\hline TOTAL & 512 & $100.00 \%$ \\
\hline
\end{tabular}

*Mansonella perstans was not confirmed using PCR technique.

From the table 2, the incidence or presence of the haemoparasites in the blood of the donors were represented in percentages. $270(52.7 \%)$ of the sample population had no infection; $121(23.6 \%)$ of the population were infected with $P$. falciparum; 69 (13.5\%) with HBsAg; 29 (5.7\%) had HCV infection; 11 (2.1\%) with P. malariae; 7 (I.4\%) had T. brucei gambiense infection; 5 (1\%) had microfilaria infection, $4(0.8 \%)$ of the $1 \%$ was unsheathed, identified to be $M$. perstans, while $1(0.2 \%)$ were sheathed and identified to be $L$. loa (was however not confirmed using PCR techniques).

The parasite intensity among the donors is recorded in table 1 . The most intense parasitic infection is $P$. falciparum with the highest incidence and $L . l o a$ is the least intense infection.

Table 3: Presence of Parasites In Relation To Donor's Occupation

\begin{tabular}{lllllll}
\hline PARASITES & H/W & FARMERS & ARTISANS & STUDENTS & C/S & TOTAL \\
\hline P. falciparum & 7 & 70 & 19 & 10 & 15 & 121 \\
P. malariae & 0 & 9 & 0 & 0 & 2 & 11 \\
HBsAg & 1 & 40 & 8 & 12 & 8 & 69 \\
HCV & 1 & 19 & 3 & 4 & 2 & 29 \\
T. brucei gambiense & 0 & 7 & 0 & 0 & 0 & 7 \\
M. perstans & 1 & 3 & 0 & 0 & 0 & 4 \\
L. loa & 0 & 1 & 0 & $26 / 26.0 \%$ & $27 / 32.5 \%$ & 242 \\
Total No. Infected/ (\%) & $10 / 83.3 \%$ & $149 / 66.8 \%$ & $30 / 31.9 \%$ & 100 & 83 & 512 \\
Total No. Sampled & 12 & 223 & 94 & & &
\end{tabular}

$\mathrm{H} / \mathrm{W}=$ Housewives

$\mathrm{C} / \mathrm{S}=$ Civil servants

Table 3 shows the haemoparasitic infection in relation to the occupation of the donors.

The total number of each occupation type that had any form of haemoparasitic infection was compared with the total number of donors that were encountered in that occupation type. This relationship, showed housewives to be the highest susceptible group and artisans the lowest.

Table 4: Relation of the Presence of Parasites to Location of Donor Recruitment

\begin{tabular}{|c|c|c|c|c|c|c|c|c|c|}
\hline \multirow{2}{*}{$\frac{\text { PARASITES }}{P \text {. falciparum }}$} & \multicolumn{5}{|c|}{ JENGRE RIYOM RANTIYA BUKURU GAINJI } & \multicolumn{2}{|c|}{ ANGWARE JETS } & \multicolumn{2}{|c|}{ FECOLART D/KOWA } \\
\hline & 32 & 9 & 1 & 15 & 19 & 10 & 8 & 5 & 10 \\
\hline P. malariae & 4 & 2 & 0 & 1 & 0 & 2 & 1 & 0 & 1 \\
\hline HBsAg & 15 & 2 & 3 & 8 & 9 & 5 & 13 & 5 & 7 \\
\hline $\mathrm{HCV}$ & 9 & 2 & 2 & 2 & 2 & 4 & 4 & 1 & 3 \\
\hline T. brucei gambiense & 5 & 1 & 1 & 0 & 0 & 0 & 0 & 0 & 0 \\
\hline $\begin{array}{l}\text { M. perstans } \\
\text { (unsheathed } \\
\text { microfilaria) }\end{array}$ & 4 & 0 & 0 & 0 & 0 & 0 & 0 & 0 & 0 \\
\hline $\begin{array}{l}L . \quad \text { loa (sheathed } \\
\text { microfilaria) }\end{array}$ & & 0 & 0 & 0 & 0 & 0 & 0 & 0 & 0 \\
\hline No. INFECTED/ (\%) & $70 / 61.9 \%$ & $16 / 72.7$ & $7 / 29.2 \%$ & $26 / 55.3 \%$ & $30 / 65.2 \%$ & $021 / 43.8 \%$ & $26 / 27$ & $11 / 23.4 \%$ & $21 / 30.0 \%$ \\
\hline No. SAMPLED & 113 & 22 & 24 & 47 & 46 & 48 & 95 & 47 & 70 \\
\hline
\end{tabular}

JETS= Jos ECWA Theological Seminary

FECOLART $=$ Federal College of Agricultural Resources Technology

D/KOWA= Dadin Kowa

Table 4 shows the haemoparasitic infection in relation to the location of the donors recruited. The total number 
of donors from each location that had any form of haemoparasitic infection was compared with the total number of donors that were recruited from that particular location.

This relationship, showed Riyom to be the highest susceptible location and FECOLART the lowest in terms of haemoparasitic infection.

\section{Discussion}

The survey of the presence of haemoparasites in apparently healthy donors is very important in the context of transfusion biosafety. Infected recipients may be asymptomatic, hence carriers and reservoirs for further transmission; or symptomatic, exhibiting the various clinical manifestations, associated with these haemoparasitic infections [7].

Blood transfusion is an important intervention in hospitals and health centres, but it also represents a route for transmission of pathogens of the blood such as parasites, bacteria and viruses. It is however important to note that haemoparasitic infectivity reduces with time, in the case or stored blood outside the host [8].

Therefore those in quest for fresh blood are the most liable victims of blood transfusion related haemoparasitic infections [8]. The highest incidence was seen to be in Plasmodium infection. The two species encountered were the $P$. falciparum and P. malariae infection, both of which are endemic in Nigeria [9].

All Plasmodium transfusion routes have been identified. This includes blood donation. These donors are apparently healthy and asymptomatic, hence the willingness to donate. This asymptomatic presentation may be due to the development of the immunity to malaria infection, which comes with their age, since most of the donors are adults [10].

The acquired immunity of the potential donors reduces the frequency of the malaria infection, and its associated symptoms as mostly observed with adults (donor category), unlike that seen in children [10].

On world malaria day it was reported that though malaria burden has reduced by about $60 \%$ since 2000 , it will be very difficult to eliminate malaria infection. This suggested difficulty is substantiated by factors like transfusion related acquisition of malaria infection [7].

The incidence of Plasmodium falciparum was followed by the incidence of the two types of viruses encountered. Though broadly speaking, they can be considered as parasites, they are however viruses. However, a few things must be said since they are very important in blood biosafety. The presence of one or both of these parasites leads to acute or chronic hepatitis, which inflames and damage the liver [11].

These viruses are most times only reduced to a non detectable viral load. A complete cure is rare, making it better avoided than managed or cured. Also both types of hepatitis viruses are almost a $100 \%$ transmissible by blood transfusion [11].

All the donors infected with trypanosomes were farmers. This could be due to human interaction with the vector resting sites, which exposed them to the infection. More research is needed on significance in blood donation since the African trypanosomiasis is said to be only vector transmitted [4, 12].

However, the risk of vector transmission is a thing of concern if this research was done in another light.

The presence of $M$. perstans and L. loa was significant. The age mostly infected in this study (33-45), is of high socioeconomic importance because this is the productive age. Microfilariae are also dangerous in transfusion since it is capable of eliciting very serious transfusion reactions, which can lead to death, especially if the patient or recipient is vulnerable. Mansonella perstans and $L$. loa have also been found to coexist in areas where they are found [13].

In a study in the north-western zone of Bauchi State [14] reported $0.7 \%$ and $1.4 \%$ prevalence of $L$. loa and $M$. perstans, respectively. In practice, there are transfusion reactions of unknown origin. This was a major reason for this study. From previous studies, microfilariae infections have been pinpointed as the cause of mortality in many blood transfusion cases. This is due to reactions, which turn out to be very severe, especially in those with some sort of challenge immunologically (immuno-suppressed or immuno-compromised). These transfused microfilariae circulates in the recipient's blood, without developing into adult worms, but sufficient damage is done by the larva found in circulation, migrating into wherever it may, even into organs [15]. This therefore makes the fact that microfilariae were encountered in this study, very important.

All blood group types were observed to be susceptible to parasitaemia. Some blood groups turned out to be quite high, but when related to the turnout of individuals within the blood group type, the explanation became clearer that parasitaemia cannot be judged to be high just from this statistical analysis. However, more work can be done by taking equal number of blood group types. This implies that while screening for blood parasites, all donors should have equal treatment, irrespective of the blood type (WHO, 2002). The highest incidence was seen to be in Plasmodium infection. The two species encountered were the $P$. falciparum and $P$. malariae infection, both of which are encountered in Nigeria [9].

This is important to note since though malaria infection is greatly reduced due to the use of long lasting insecticide treated nets and the various Artemisinin combination treatments, malaria still kills up to 2000 people daily in Africa, especially children [7]. 
The work also showed the spread of the haemoparasitic infection depending on the occupation of the donors and the various locations of donor recruitment. This is to be investigated further, for a more meaningful discussion in these areas. It can however be said that more rural settlements had more infection than the more urban settlements. Predisposing factors will be further investigated. Also, the elites had lesser infection than the housewives and farmers. Also, this is an opening for further investigation.

\section{Conclusion}

This study reveals that the infection rate of the haemoparasites differs, as well as parasite intensity. The highest infection occurred due to $P$. falciparum, with an infection rate of as high as $23.6 \%$ while the lowest infection was due to $L$. loa, with an infection rate of $0.2 \%$. It was observed that parasitaemia was possible, regardless of the blood group type.

This study therefore concludes and stresses the need to take parasites associated with blood transfusion very seriously, considering the dangers of doing otherwise. There could be evidence and a possibility of evolutionary mechanisms of attenuation of symptoms of haemoparasitic infections in humans.

Also it is important to note that the people in need of blood transfusion in very many cases may not have all it takes to withstand invasion of these parasite. With this in mind, it is expedient to ensure that the necessary precautions are taken to avoid fatal consequences. Therefore, This study contribute to knowledge in the following ways; according to the criteria given by World health Organization in 2010 (16) Plasmodium falciparum, Plasmodium malariae, Trypanosoma brucei gambiense, Mansonella perstans (unsheathed microfilaria), loa loa (sheathed microfilaria) were not included in the mandatory minimum requirement for screening of blood donor for infection. With our findings, it is necessary that it should be included because of Current and emerging infectious risks of blood transfusion $(17,18)$.

\section{Recommendations}

Following the findings, the following recommendations are tendered:

1. Proper screening of blood for all haemoparasites before blood is transfused to recipient should be carried out.

2. Recipients may be asymptomatic after contracting a post transfusion infection, hence carriers may constitute a reservoir for parasites, further maintaining transmission. This calls for the need for an after transfusion screening and documentation of the risk of transfusion related haemoparasitic infections.

\section{Conflicts of Interest}

There is no conflict of interest.

\section{References}

1. World Health Assembly resolution (1975). Utilization and supply of human blood and blood products. Geneva, World Health Organization. WHA28.72.

2. World Health Assembly resolution (2005). Proposal to establish World Blood Donor Day. Geneva, World Health Organization. WHA58. 13

3. World Health Organization (2001-2002). Blood transfusion safety. Global database on blood safety: report.www.who.int/blood safety/GDBS report..

4. World Health Organization (2008). Blood Safety and Availability fact sheet 2013....2008 CD142. R5 Blood...www.swisstransfusion.ch/_pdf/Neclam.

5. Dodd, R. Y. (2007). Current risk for transfusion-transmitted infections. Current Opinion in Hematology, 14(6), 671-676.

6. Cheesebrough, M. (1998): Parasitological tests. vol.3.2 $2^{\text {nd }}$ ed., Medical Laboratory Manual for Tropical Countries. Cambridge, United Kingdom: Tropical Health Technology, 239- 295.

7. World Health Organization (2016). World Malaria day report. Eliminating-Malaria Push. Malaria Journal 2016 15:303.

8. .Schmidt, M., Geilenkeuser W. J., Sireis, W., Seifried E. and K. Hourfar (2014). Emerging Pathogens- How Safe is Blood? Transfusion Medicine Haemotherapy, 41:10-17.

9. .,Ajayi, O. O., Ajayi, O. A., Turshak, L. G. and Dakyahas. J.Y. (2014). Prevalence of Congenital malaria in Jos, Plateau State. African Journal Online, 12(1).

10. Ikeh E. I. and Teclaire N. N. (2008). Prevalence of Malaria Parasitaemia and Associated Factors in Febrile under 5- Children seen in PHC centres in Jos, North Central area. Nigeria Post graduate Medical Journal, 15(2), 05-09.

11. Kostritsa, S. S., Kuzin, S. N,..Khukhlovich, P. A. Shakhgil'dian, I. V. Doroshenko, N. V. Blinov, V. M

12. Loparen, V. N Sukhanova, L. L Gol'tsov, V. A . Drobeniuk, Z. A et al., (1994). The detection of antibodies to the hepatitis $\mathrm{C}$ virus and to HBsAg in blood donors with viral hepatitis and cancerous diseases on the 
republic of Moldova. Voprosy Virusologii, 39(2), 93-94.

13. Uba. B. V (2012). Knowledge and Prevalence of Human African Trypanosomiasis among Residents of Kachia grazing reserve Kaduna State. Accessed 5-15-16, 3:34pm.

14. .Agbolade O. M, and. Akinboye D.O. (2005). Detection of microfilariae with counting chamber technique in some Nigerian rural communities. African Journal of Biotechnology, 4: 367- 370.

15. Anosike, J. C., Nwoke, B.E.B., Onwiliri, C.O.E., Obiukwu, C.E., Duru, A. F., Nwachukwu, M. I., Ukaga, C. N., Uwaezuoke, J. C., Uduji, O. S.,. Amajuoyi, O. U. and Nkem B.I. (2004). Prevalence of parasitic diseases among nomadic Fulanis of south-eastern Nigeria. Annals of Agriculture and Environmental Medicine, 11: 221- 225.

16. Choudhury, N., Murthy, P. K.. Chatterjee, R. K., Khan M.A. And Ayyagari A. (2003). Transmission of filarial infection through blood transfusion. Indian Journal of Pathological Microbiology,46(3), 367-370.

17. Screening donated blood for transfusion-transmissible infections. Geneva, World Health Organization, 2010. (http://www.who.int/bloodsafety/publications/bts screendondbloodtransf/en/index.html accessed on 17 August 2012).

18. Alter H. Emerging, re-emerging and submerging infectious threats to the blood supply. Vox Sanguinis, 2004 , 87(Suppl. s2):56-61.

19. Busch, M, and Kleinman, S.N.( 2003). Current and emerging infectious risks of blood transfusion. Journal of the American Medical Association, , 289(8):959-962. 